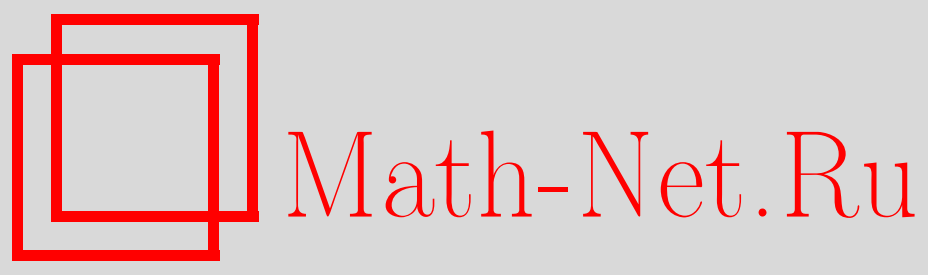

А. И. Даниленко, В. В. Рыжиков, Спектральные кратности преобразований, сохраняющих бесконечную меру, Функи. анализ и его прил., 2010, том 44, выпуск 3, 1-13

DOI: https://doi.org/10.4213/faa3003

Использование Общероссийского математического портала MathNet.Ru подразумевает, что вы прочитали и согласны с пользовательским соглашением

http://www . mathnet.ru/rus/agreement

Параметры загрузки:

IP : 3.81 .55 .215

26 апреля 2023 г., 17:29:33

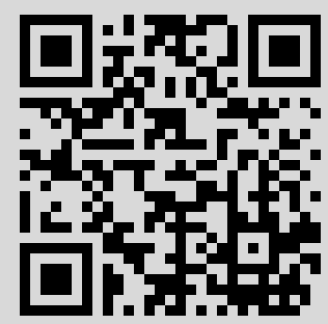


Функционалъный анализ и его приложения

2010, т. 44, вып. 3, с. 1-13

УДК 517.987

\title{
Спектральные кратности преобразований, сохраняющих бесконечную меру*
}

\author{
(c) 2010. А. И. ДАниленко, В. В. Рыжиков
}

\begin{abstract}
Любое непустое подмножество $E$ натурального ряда $\mathbb{N}$ реализовано как множество кратностей спектра купмановского оператора, отвечающего эргодическому консервативному преобразованию, сохраняющему бесконечную меру.
\end{abstract}

\section{Введение}

Пусть $T$ - эргодическое консервативное обратимое преобразование стандартного измеримого пространства $(X, \mathfrak{B}, \mu)$, сохраняющее $\sigma$-конечную меру $\mu$. Ему отвечает унитарный купмановский оператор $U_{T}$ в гильбертовом пространстве $L^{2}(X, \mu)$ :

$$
U_{T} f:=f \circ T .
$$

В случае конечной меры $\mu$ действие оператора $U_{T}$ обычно рассматривается только на ортогональном дополнении к пространству постоянных функций.

Общий вопрос спектральной теории динамических систем:

$$
\text { какие унитарные операторы являются купмановскими? }
$$

Хорошо известны такие частные случаи проблемы (0.1) для конечной меры $\mu$, как задача Банаха о простом лебеговском спектре, задача Колмогорова о групповом свойстве спектра (см. [6], [28]), задача Рохлина об однородном спектре и более общий вопрос о множестве спектральных кратностей. Этот вопрос формулируется следующим образом:

какие подмножества множества $\mathbb{N}$

реализуемы как $\mathscr{M}(T)$ для эргодического T?

Здесь $\mathscr{M}(T)$ обозначает множество существенных значений функции спектральной кратности для оператора $U_{T}$. Несмотря на значительный прогресс, достигнутый в работах [3]-[5], [8], [10], [14], [18]-[26] и др., этот давний вопрос спектральной теории динамических систем с конечной инвариантной мерой остается открытым.

В настоящей статье вопрос (0.2) рассматривается в классе эргодических консервативных преобразований, сохраняющих бесконечную меру, и в этом классе получен полный ответ на него.

Теорема 0.1. Для любого непустого множества $E \subset \mathbb{N}$ найдется эргодическое сохраняющее бесконечную меру преобразование $T$ со свойством кратной рекуррентности, для которого $\mathscr{M}(T)=E$.

*Работа второго из авторов частично поддержана грантами НШ 3038.2008.1 и НШ 8508.2010.1. 
Пример такого преобразования в случае, когда при $p>0$ число $p+1$ лежит в $E$, мы ищем в виде $T^{\odot p} \times T_{\alpha}$, где $T^{\odot p}-$ симметрическая степень подходящего преобразования $T$, а $T_{\alpha}$ - некоторое его компактное расширение. Если $E=$ $\{p+1\}$, то примером служит произведение $T^{\odot p} \times T$. Для доказательства теоремы мы также модифицируем для случая бесконечных мер технику недавней работы [10] (которая, в свою очередь, использует методы большинства из упомянутых статей), развитую для систем, действующих на вероятностном пространстве.

В случае бесконечной меры обнаруживаются специфические явления, не имеющие места в случае вероятностной меры. Например, эргодичность не является спектральным свойством. K тому же нет хорошего определения слабого перемешивания. Действительно, для любого $p>0$ найдется преобразование $T$, такое, что его $p$-я декартова степень $T^{\times p}$ эргодична, а степень $T^{\times(p+1)}$ нет [17]. Напомним, что преобразование $T$ на $(X, \mu)$ называется кратно рекуррентным преобразованием, если для любого $A \subset X$ положительной меры и любого $p>0$ найдется число $k>0$, такое, что $\mu\left(A \cap T^{k} A \cap \cdots \cap T^{k p} A\right)>0$. Это понятие является естественным обобщением понятия консервативности (которая соответствует случаю $p=1)$. Фюрстенберг показал, что при $\mu(X)<\infty$ каждое $\mu$-сохраняющее преобразование обладает свойством кратной рекуррентности [13]. Однако для бесконечных пространств известны эргодические преобразования (такие, что и все их декартовы степени эргодичны), которые не обладают свойством кратной рекуррентности [2]. Информацию об этих и других контрпримерах читатель найдет в [1], [9], [12].

Причина, по которой доказательство теоремы 1 не переносится на случай вероятностной меры, состоит в том, что у сомножителей $T^{\odot p}$ и $T_{\alpha}$ в произведении $T^{\odot p} \times T_{\alpha}$ в вероятностном случае появляются инвариантные одномерные пространства констант, которые добавляют лишние кратности.

\section{$\S 1$. Задача Рохлина об однородном спектре для преобразований, сохраняющих бесконечную меру}

Задача Рохлина формулируется следующим образом:

найдется ли для заданного $n>1$ эргодическое преобразование с однородным спектром кратности $n$ ?

Это частный случай проблемы (0.1), и ответ на поставленный здесь вопрос играет важную роль в доказательстве теоремы 0.1. Отметим, что в случае конечной меры задача Рохлина решена в [3], [24] для $n=2$, а для произвольных $n-$ в [4] и конструктивно в [8]. Чтобы решить задачу об однородном спектре для преобразований пространства с бесконечной мерой, достаточно рассмотреть естественные факторы их декартовых степеней (см. [18], [5]).

Лемма 1.1. Пусть $\Gamma-$ подгруппа группь перестановок $\mathfrak{S}_{k}$. Пусть $\mathrm{V}-$ унитарный оператор с простым непрерывным спектром, причем спектр оператора $V^{\odot k}$ является простым. Тогда ограничение $V_{\Gamma}^{\otimes k}$ оператора $V^{\otimes k}$ на подпространство Г-инвариантных тензоров имеет однородный спектр кратности $k ! / \# \Gamma$.

Доказательство. Пусть $\sigma$ - мера максимального спектрального типа оператора $V$ и $\pi: \mathbb{T}^{k} \rightarrow \mathbb{T}-$ гомоморфизм, заданный равенством $\pi\left(z_{1}, \ldots, z_{k}\right)=$ $z_{1} \cdots z_{k}$.

Будем считать, что оператор $V$ является оператором умножения на переменную $z, z \in \mathbb{T}$, в пространстве $L^{2}(\mathbb{T}, \sigma)$. Так как мера $\sigma$ непрерывна, группа 
$\mathfrak{S}_{k}$ действует свободно на почти всех слоях $\pi^{-1}(z)$, оснащенных условной мерой $\sigma^{k} \mid \pi^{-1}(z)$. Оператор $V^{\odot k}$ имеет простой спектр; следовательно, почти все условные меры сосредоточены в $k$ ! точках положительной меры (набор точек зависит от слоя). Действие группы $Г$ на слое разбивает это множество точек на $k ! / \# \Gamma$ орбит одинаковой мощности. Таким образом, оператор $V_{\Gamma}^{\otimes k}$ унитарно эквивалентен прямой сумме $k ! / \# \Gamma$ копий оператора $V^{\odot} k$, причем каждая копия имеет простой спектр.

Следствие 1.2. Пусть $T$ - эргодическое консервативное преобразование, сохраняющее бесконечную меру, причем оператор $U_{T}^{\odot k}$ имеет простой спектр. Тогда при $k>1$ выполняется равенство $\mathscr{M}\left(T^{\odot(k-1)} \times T\right)=\{k\}$.

Доказательство. Так как преобразование $T$ действует в пространстве с бесконечной мерой, оператор $U_{T}$ обладает непрерывным спектром. Осталось заметить, что $U_{T \odot(k-1) \times T}=U_{T}^{\odot(k-1)} \otimes U_{T}=\left(U_{T}\right)_{\mathfrak{S}_{k-1}}^{\otimes k}$, и применить лемму 1.1 .

Отметим, что следствие 1.2 пока не дает решения задачи Рохлина, так как не ясно, будет ли преобразование $U_{T \odot(k-1)} \times T$ эргодическим. Требуемый пример будет построен в ходе доказательства теоремы 0.1.

\section{§2. Предварительные сведения и обозначения}

Пусть $G$ - счетная абелева группа, $H$ - ее подгруппа, $v: G \rightarrow G$ - групповой автоморфизм, а $\widehat{G}$ и $\widehat{v}$ обозначают дуальную группу к $G$ и дуальный автоморфизм к $v$ соответственно. Через \# $A$ обозначается мощность множества $A$. Положим

$$
\begin{aligned}
M_{v}^{h} & :=\#\left(\left\{v^{i}(h) \mid i \in \mathbb{Z}\right\} \cap H\right), \\
L(G, H, v) & :=\left\{M_{h}^{v} \mid h \in H \backslash\{0\}\right\}, \\
\mathscr{G} & :=\left\{a \in \widehat{G} \mid \exists p>0 \widehat{v}^{p}(a)=a\right\}, \\
l_{g}(a) & :=\frac{1}{p} \sum_{i=0}^{p-1} a\left(v^{i}(g)\right) \quad \text { для всех } a \in \mathscr{G}, \text { таких, что } \widehat{v}^{p}(a)=a, g \in G .
\end{aligned}
$$

В этом параграфе мы приводим два вспомогательных утверждения, играющих ключевую роль при подсчете кратностей спектра изучаемых преобразований.

Лемма 2.1 (см. [10]). Для заданного произвольного множества $E \subset \mathbb{N}$ найдутся счетная абелева группа $G$, подгруппа $H \subset G$ и автоморфизм $v: G \rightarrow G$, такие, что $E=L(G, H, v)$. Кроме того, выполняются следующие условия:

(i) nодгруппа $\mathscr{G}$ счетна и плотна в $\widehat{G}$;

(ii) $\#\left\{l_{h}(a) \mid a \in \mathscr{G}\right\}=\infty$ для любого $h \in H \backslash\{0\}$;

(iii) если $g_{1}, g_{2} \in G u v^{i}\left(g_{1}\right) \neq g_{2}$ для всех $i \in \mathbb{Z}$, то найдется $a \in \mathscr{G}$, такое, чmo $l_{g_{1}}(a) \neq l_{g_{2}}(a)$.

Следующая лемма доказана в [19] при несколько более сильных условиях. Мы даем другое, более короткое доказательство.

Лемма 2.2. Пусть $V$ и $W$ - унитарные операторы с простым спектром, действующие в гильбертовых пространствах $\mathscr{H}$ и $\widetilde{\mathscr{H}}$ соответственно. Предположим, что для каждого $i=1, \ldots, k$ найдутся последовательности $n_{t}^{(i)} \rightarrow \infty$, 
$m_{t}^{(i)} \rightarrow \infty$ и три комплексных числа $\kappa_{i}, \widetilde{\kappa}_{i}, \widetilde{\kappa}_{i} \neq \kappa_{i}, u \delta_{i}$, такие, что иметот место слабые сходимости

(i) $V^{n_{t}^{(i)}} \rightarrow \kappa_{i} I+\delta_{i} V^{*}, W^{n_{t}^{(i)}} \rightarrow \kappa_{i} I+\delta_{i} W^{*}$;

(ii) $V^{m_{t}^{(i)}} \rightarrow \kappa_{i} I+\delta_{i} V^{*}, W^{m_{t}^{(i)}} \rightarrow \widetilde{\kappa}_{i} I+\delta_{i} W^{*}$.

Предположим, кроме того, что все числа $\kappa_{i} / \delta_{i}$ различны. Тогда $V^{\odot k} \otimes W$ имеет простой спектр.

Доказательство. Без ограничения общности можно считать, что $\mathscr{H}=$ $L^{2}\left(\mathbb{T}, \sigma_{V}\right), \widetilde{\mathscr{H}}=L^{2}\left(\mathbb{T}, \sigma_{W}\right)$ и $V f(z)=z f(z), W g(z)=z g(z)$ для всех $f \in \mathscr{H}$, $g \in \widetilde{\mathscr{H}}, z \in \mathbb{T}$. Здесь $\sigma_{V}$ и $\sigma_{W}$ обозначают меры максимального спектрального типа для операторов $V$ и $W$ соответственно. Имеем

$$
\begin{gathered}
\mathscr{H}^{\odot k} \otimes \widetilde{\mathscr{H}}=L_{\mathrm{sym}}^{2}\left(\mathbb{T}^{k}, \sigma_{V}^{k}\right) \otimes L^{2}\left(\mathbb{T}, \sigma_{W}\right), \\
\left(V^{\odot k} \otimes W\right) f\left(z_{1}, \ldots, z_{k}, z\right)=z_{1} \cdots z_{k} z f\left(z_{1}, \ldots, z_{k}, z\right)
\end{gathered}
$$

для всех $f \in \mathscr{H}^{\odot k} \otimes \widetilde{\mathscr{H}}$.

Пусть $\mathscr{A}$ - алгебра фон Неймана, порожденная оператором $V^{\odot k} \otimes W$. Мы рассматриваем элементы из $\mathscr{A}$ как ограниченные функции на $\mathbb{T}^{k+1}$, инвариантные относительно перестановок первых $k$ координат. Из (i) и (ii) получаем, что обе функции

$$
\begin{aligned}
& \left(z_{1}, \ldots, z_{k}, z\right) \mapsto\left(\kappa_{i}+\delta_{i} z\right) \cdot \prod_{l=1}^{k}\left(\kappa_{i}+\delta_{i} z_{l}\right), \\
& \left(z_{1}, \ldots, z_{k}, z\right) \mapsto\left(\widetilde{\kappa}_{i}+\delta_{i} z\right) \cdot \prod_{l=1}^{k}\left(\kappa_{i}+\delta_{i} z_{l}\right)
\end{aligned}
$$

принадлежат $\mathscr{A}$ для каждого $i=1, \ldots, k$. Так как $\kappa_{i} \neq \widetilde{\kappa}_{i}$, то алгебре $\mathscr{A}$ принадлежат функции

$$
\left(z_{1}, \ldots, z_{k}, z\right) \mapsto \prod_{l=1}^{k}\left(\frac{\kappa_{i}}{\delta_{i}}+z_{l}\right)=\sum_{l=0}^{k}\left(\frac{\kappa_{i}}{\delta_{i}}\right)^{l} P_{l}\left(z_{1}, \ldots, z_{k}\right),
$$

где $P_{l}\left(z_{1}, \ldots, z_{k}\right)$ - основные симметрические многочлены, $i=1, \ldots, k$. Следовательно, многочлены $P_{0}, \ldots, P_{k}$ также принадлежат алгебре $\mathscr{A}$ (по свойству детерминанта Вандермонда и условию леммы). Но $P_{0}, \ldots, P_{k}$ порождают алгебру всех симметрических многочленов от $k$ переменных. Обозначим через $\mathscr{Z}$ $\left(V^{\odot k} \otimes W\right)$-циклическое пространство, порожденное постоянной функцией $1 \in$ $\mathscr{H}^{\odot k} \otimes \widetilde{\mathscr{H}}$. Поскольку $\mathscr{Z}$ инвариантно относительно $\mathscr{A}$ и линейное подпространство $\mathscr{P}_{\text {sym }}(k) 1$ плотно в $L_{\text {sym }}^{2}\left(\mathbb{T}^{k}, \sigma_{V}^{k}\right)$, получаем, что $\mathscr{Z} \supset L_{\text {sym }}^{2}\left(\mathbb{T}^{k}, \sigma_{V}^{k}\right) \otimes 1$ и, следовательно,

$$
\mathscr{Z} \supset L_{\mathrm{sym}}^{2}\left(\mathbb{T}^{k}, \sigma_{V}^{k}\right) \otimes z^{n} \text {. }
$$

Это приводит к равенству $\mathscr{Z}=L_{\text {sym }}^{2}\left(\mathbb{T}^{k}, \sigma_{V}^{k}\right) \otimes L^{2}\left(\mathbb{T}, \sigma_{W}\right)$.

Пусть $E$ - подмножество в $\mathbb{N}$. Переходя к объектам, дуальным к группе, подгруппе и автоморфизму в утверждении леммы 2.1, получаем компактную метризуемую абелеву группу $K$, ее замкнутую подгруппу $H$ и непрерывный автоморфизм $v$ группы $K$, такие, что

$$
E=L(\widehat{K}, \widehat{K / H}, \widehat{v})
$$


причем выполняются условия (i)-(iii) леммы 2.1. Подмножество $v$-периодических точек в $K$ обозначим через $\mathscr{K}$ и будем придерживаться этого обозначения до конца работы.

Пусть $T$ - некоторое эргодическое преобразование стандартного измеримого пространства $(X, \mu)$, а $\mathscr{R} \subset X \times X$ - отношение $T$-орбитальной эквивалентности. Измеримое отображение $\alpha$ из $\mathscr{R}$ в $K$ называется коциклом эквивалентности $\mathscr{R}$, если

$$
\alpha(x, y) \alpha(y, z)=\alpha(x, z) \quad \text { для всех }(x, y),(y, z) \in \mathscr{R} .
$$

Коциклы $\alpha, \beta: \mathscr{R} \rightarrow K$ когомологичны, если существуют измеримое отображение $\phi: X \rightarrow K$ и множество полной меры $B \subset X$, такие, что для всех $(x, y) \in \mathscr{R} \cap B \times B$

$$
\alpha(x, y)=\phi(x) \beta(x, y) \phi(y)^{-1} .
$$

Если преобразование $S$ коммутирует с $T$ (т. е. $S$ принадлежит $C(T)$, централизатору преобразования $T$ в группе всех сохраняющих меру преобразований), то коцикл $\alpha \circ S: \mathscr{R} \rightarrow K$ корректно определен равенством $(\alpha \circ S)(x, y):=$ $\alpha(S x, S y)$.

Пусть $\lambda_{K / H}$ - мера Хаара на $K / H$. Обозначим через $T_{\alpha, H}$ следующее преобразование пространства $\left(X \times K / H, \lambda_{K / H}\right)$ :

$$
T_{\alpha, H}(x, k+H):=(T x, \alpha(T x, x)+k+H) .
$$

Это преобразование является косым произведением над $T$. Для краткости $T_{\alpha,\{0\}}$ обозначается через $T_{\alpha}$. Как и случае конечной меры, имеет место разложение оператора $U_{T_{\alpha, H}}$ в ортогональную сумму, $U_{T_{\alpha, H}}=\bigoplus_{\chi \in \widehat{K / H}} U_{T, \chi}$, где $U_{T, \chi}-$ унитарный оператор в $L^{2}(X, \mu)$, определенный равенством

$$
U_{T, \chi} f(x)=\chi(\alpha(T x, x)) f(T x), \quad x \in X .
$$

Замечание 2.3. Непосредственно проверяется следующее утверждение: если для некоторого $S \in C(T)$

$\alpha \circ S$ когомологичен $v \circ \alpha$, то $U_{T, \chi}$ и $U_{T, \widehat{v}^{i}(\chi)}$ унитарно эквивалентны для всех $i \in \mathbb{Z}$.

\section{§3. Конструкция базисного преобразования и коцикла}

Для доказательства теоремы 0.1 мы воспользуемся $(C, F)$-конструкцией (см. [16], [7]-[9]). Предварительно дадим ее общее определение. Пусть заданы последовательности $\left(C_{n}\right)_{n>0}$ и $\left(F_{n}\right)_{n \geqslant 0}$ конечных подмножеств множества $\mathbb{Z}$, причем

- $F_{n}=\left\{0,1, \ldots, h_{n}-1\right\}, h_{0}=1, \# C_{n}>1,0 \in C_{n}$,

- $F_{n}+C_{n+1} \subset F_{n+1}$,

- $\left(F_{n}+c\right) \cap\left(F_{n}+c^{\prime}\right)=\varnothing$, если $c \neq c^{\prime}, c, c^{\prime} \in C_{n+1}$,

- $\lim _{n \rightarrow \infty} h_{n} /\left(\# C_{1} \cdots \# C_{n}\right)=\infty$.

Пусть $X_{n}:=F_{n} \times C_{n+1} \times C_{n+2} \times \cdots$. Наделяем это множество компактной польской топологией прямого произведения. Отображение

$$
\left(f_{n}, c_{n+1}, c_{n+2}\right) \mapsto\left(f_{n}+c_{n+1}, c_{n+2}, \ldots\right)
$$

является топологическим вложением пространства $X_{n}$ в $X_{n+1}$. Теперь положим $X:=\bigcup_{n \geqslant 0} X_{n}$, оснащая $X$ (локально компактной польской) топологией индуктивного предела. При заданном $A \subset F_{n}$ обозначим через $[A]_{n}$ цилиндр 
$\left\{x=\left(f, c_{n+1}, \ldots,\right) \in X_{n} \mid f \in A\right\}$. Тогда семейство $\left\{[A]_{n} \mid A \subset F_{n}, n>0\right\}$ всех компактных открытых подмножеств в $X$ является базой топологии на $X$. Обозначим через $\mathfrak{B}$ борелевскую $\sigma$-алгебру, порожденную этой топологией.

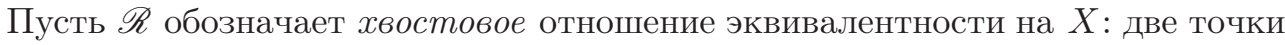
$x, x^{\prime} \in X$ являются $\mathscr{R}$-эквивалентными, если найдется $n>0$, такое, что

$$
x=\left(f_{n}, c_{n+1}, \ldots\right) \in X_{n}, \quad x^{\prime}=\left(f_{n}^{\prime}, c_{n+1}^{\prime}, \ldots\right) \in X_{n}
$$

и $c_{m}=c_{m}^{\prime}$ для всех $m>n$. Напомним, что мера на $(X, \mathfrak{B})$ является $\mathscr{R}$-инвариантной, если она инвариантна относительно любого измеримого преобразования $S: X \rightarrow X$, график которого содержится в $\mathscr{R}$. Мера $\mu$, являющаяся $\mathscr{R}$-инвариантной, называется $\mathscr{R}$-эргодичной, если каждое $\mathscr{R}$-насыщенное подмножество множества $X$ имеет полную или нулевую $\mu$-меру. Существует единственная неатомическая борелевская $\sigma$-конечная мера $\mu$ на $X$, инвариантная и эргодическая относительно $\mathscr{R}$, такая, что $\mu\left(X_{0}\right)=1$.

Определим преобразование $T$ на $(X, \mu)$, полагая

$$
T\left(f_{n}, c_{n+1}, \ldots\right):=\left(1+f_{n}, c_{n+1}, \ldots\right) \text { при } f_{n}<h_{n}-1, n>0 .
$$

Эта формула определяет $T$ на $X_{n}$. Устремляя $n$ к бесконечности, мы определяем отображение $T$ на всем пространстве $X$ (без счетного числа точек). Это отображение сохраняет меру $\mu$. Более того, отношение $T$-орбитальной эквивалентности совпадает с $\mathscr{R}$ (на множестве, где $T$ определено). Преобразование $T$ будем называть $(C, F)$-преобразованием, ассоциированным с $\left(C_{n+1}, F_{n}\right)_{n \geqslant 0}$.

Напомним понятие $(C, F)$-коцикла (см. [8]). Пусть задана последовательность отображений $\alpha_{n}: C_{n} \rightarrow K, n=1,2, \ldots$ Определим борелевский коцикл $\alpha: \mathscr{R} \cap\left(X_{0} \times X_{0}\right) \rightarrow K$, положив

$$
\alpha\left(x, x^{\prime}\right):=\sum_{n>0}\left(\alpha_{n}\left(c_{n}\right)-\alpha_{n}\left(c_{n}^{\prime}\right)\right)
$$

для $x=\left(0, c_{1}, c_{2}, \ldots\right) \in X_{0}, x^{\prime}=\left(0, c_{1}^{\prime}, c_{2}^{\prime}, \ldots\right) \in X_{0},\left(x, x^{\prime}\right) \in \mathscr{R}$. Чтобы определить коцикл $\alpha$ на всем $\mathscr{R}$, предварительно введем отображение $\pi: X \rightarrow X_{0}$ следующим образом. Для заданного $x \in X$ пусть $n$ является наименьшим положительным натуральным числом, для которого $x \in X_{n}$. Тогда $x=\left(f_{n}, c_{n+1}, \ldots\right)$ $\in X_{n}$. Положим

$$
\pi(x):=(\underbrace{0, \ldots, 0}_{n+1}, c_{n+1}, c_{n+2}, \ldots) \in X_{0} .
$$

Очевидно, что $(x, \pi(x)) \in \mathscr{R}$. Теперь для пар $(x, y) \in \mathscr{R}$ положим

$$
\alpha(x, y):=\alpha(\pi(x), \pi(y)) .
$$

Нетрудно проверить, что $\alpha$ есть корректно определенный коцикл отношения $\mathscr{R}$ со значениями в $K$. Мы называем его $(C, F)$-коциклом, ассоциированным $c$ $\left(\alpha_{n}\right)_{n=1}^{\infty}$.

Пусть $\bar{z}=\left(z_{n}\right)_{n=1}^{\infty}-$ некоторая последовательность натуральных чисел. Следующее утверждение является бесконечным аналогом леммы 4.11 из [8]. Важность этого утверждения для реализации требуемого набора кратностей спектра видна из замечания 2.3. 
Лемма 3.1. Пусть выполняется условие $\sum_{n=1}^{\infty} \#\left(C_{n} \triangle\left(C_{n}-z_{n}\right)\right) / \# C_{n}<\infty$. Положим

$$
X_{n}^{\bar{z}}:=\left\{0,1, \ldots, h_{n}-z_{1}-\cdots-z_{n}\right\} \times \prod_{m>n}\left(C_{m} \cap\left(C_{m}-z_{m}\right)\right) \subset X_{n} .
$$

Тогда преобразование $S_{\bar{z}}$ пространства $(X, \mu)$ корректно определено равенством

$$
S_{\bar{z}}(x):=\left(z_{1}+\cdots+z_{n}+f_{n}, z_{n+1}+c_{n+1}, z_{n+2}+c_{n+2}, \ldots\right)
$$

для всех $x=\left(f_{n}, c_{n+1}, c_{n+2}, \ldots\right) \in X_{n}^{\bar{z}}, n=1,2, \ldots$.

Более того, $S_{\bar{z}}$ коммутирует с $T$, причем $U_{T}^{z_{1}+\cdots+z_{n}}$ слабо сходится $\kappa U_{S_{\bar{z}}}$ при $n \rightarrow \infty$.

Пусть а является $(C, F)$-кочиклом, ассоциированным с последовательностью отображсений $\alpha_{n}: C_{n} \rightarrow K, n=1,2, \ldots$ Положим $C_{n}^{\circ}:=\left\{c \in C_{n} \cap\left(C_{n}-\right.\right.$ $\left.\left.z_{n}\right) \mid \alpha_{n}\left(c+z_{n}\right)=v\left(\alpha_{n}(c)\right)\right\}$. Если выполнено условие

$$
\sum_{n>0}\left(1-\# C_{n}^{\circ} / \# C_{n}\right)<\infty
$$

то коиикл $\alpha \circ S_{\bar{z}}$ когомологичен $v \circ \alpha$.

Доказательство. Проверим, что множество $D:=\bigcup_{n>1} X_{n}^{\bar{z}}$ имеет полную меру в $X$. Фиксируем $n>0$.

Предварительно отметим, что

$$
\left(X_{n}, \mu \uparrow X_{n}\right)=\left(F_{n}, \nu_{n}\right) \otimes \bigotimes_{m>n}\left(C_{m}, \kappa_{m}\right)
$$

где $\kappa_{m}$ - равнораспределенная вероятностная мера на $C_{n}$, а $\nu_{n}$ - равнораспределенная конечная мера на $F_{n}$ (см. [7]). Из леммы Бореля-Кантелли вытекает, что, с точностью до множества $\mu$-меры нуль, имеет место включение

$$
X_{n} \backslash D \subset \bigcup_{m>n}\left[\left\{h_{m}-z_{1}-\cdots-z_{m}+1, \ldots, h_{m}-1\right\}\right]_{m} .
$$

Поэтому

$$
\mu\left(X_{n} \backslash D\right) \leqslant \sum_{m>n} \frac{z_{1}+\cdots+z_{m}}{h_{m}} \mu\left(X_{m}\right)=\sum_{m>n} \frac{z_{1}+\cdots+z_{m}}{\# C_{1} \cdots \# C_{m}} \mu\left(X_{0}\right)<2 \sum_{m>n} \frac{z_{m}}{\# C_{m}} .
$$

Так как $\sum_{m>1} z_{m} / \# C_{m}<\infty$, то $\mu\left(X_{n} \backslash D\right) \rightarrow 0$ при $n \rightarrow \infty$.

Второе утверждение леммы доказывается очевидной модификацией доказательства леммы 4.11 из [8].

Теперь мы приступаем к конструкции специального $(C, F)$-преобразования и коцикла со значениями в $K$. Фиксируем разбиение

$$
\mathbb{N}=\bigsqcup_{a \in \mathscr{K}} \mathscr{N}_{a} \sqcup \bigsqcup_{k \in \mathbb{N}} \bigsqcup_{b \in \mathscr{K}} \mathscr{M}_{b, k}
$$

натурального ряда $\mathbb{N}$ на бесконечные множества. Напомним, что $\mathscr{K}$ обозначает подмножество $v$-периодических точек в $K$. Для $a \in \mathscr{K}$ число $m_{a}$ обозначает наименьший положительный период элемента $а$ под действием автоморфизма $v$. 
Определим последовательность $\left(C_{n}, h_{n}, z_{n}, \alpha_{n}\right)_{n=1}^{\infty}$ по индукции. Предположим, что эта последовательность построена до индекса $n$. Рассматриваем далее два случая.

Случай I. Пусть $n+1 \in \mathscr{N}_{a}$ для некоторого $a \in \mathscr{K}$. Положим

$$
\begin{gathered}
z_{n+1}:=2 m_{a} n h_{n}, \quad r_{n}:=n^{3} m_{a}, \\
C_{n+1}:=2 h_{n} \cdot\left\{0,1, \ldots, r_{n}-1\right\}, \\
h_{n+1}:=2 r_{n} h_{n} .
\end{gathered}
$$

Выбираем в качестве $\alpha_{n+1}: C_{n+1} \rightarrow K$ произвольное отображение, удовлетворяющее следующим условиям:

(A1) $\alpha_{n+1}\left(c+z_{n+1}\right)=v$ ○ $\alpha_{n+1}(c)$ для всех $c \in C_{n+1} \cap\left(C_{n+1}-z_{n+1}\right)$;

(A2) для всех $0 \leqslant i<m_{a}$ найдется множество $C_{n+1, i} \subset C_{n+1}$, для которого

$$
\begin{gathered}
C_{n+1, i}-2 h_{n} \subset C_{n+1}, \\
\alpha_{n+1}(c)=\alpha_{n+1}\left(c-2 h_{n}\right)+v^{i}(a) \quad \text { для всех } c \in C_{n+1, i}, \\
\left|\frac{\# C_{n+1, i}}{\# C_{n+1}}-\frac{1}{m_{a}}\right|<\frac{2}{n m_{a}} .
\end{gathered}
$$

Случай II. Пусть $n+1 \in \mathscr{M}_{b, k}$ для некоторых $b \in \mathscr{K}$ и $k \in \mathbb{N}$. Положим

$$
z_{n+1}:=m_{b} n\left(2 h_{n}(k+1)+k\right), \quad r_{n}:=n^{3}(k+1) m_{b},
$$

$D_{n+1}:=2 h_{n} \cdot\left\{0,1, \ldots, n m_{b}-1\right\} \sqcup\left(\left(2 h_{n}+1\right) \cdot\left\{1,2, \ldots, n k m_{b}\right\}+2 h_{n}\left(n m_{b}-1\right)\right)$,

$$
\begin{gathered}
C_{n+1}:=D_{n+1}+z_{n+1} \cdot\left\{0,1, \ldots, n^{2}-1\right\}, \\
h_{n+1}:=2 r_{n} h_{n}+k r_{n} /(k+1) .
\end{gathered}
$$

Выбираем в качестве $\alpha_{n+1}: C_{n+1} \rightarrow K$ отображение, удовлетворяющее следующим условиям:

(А3) $\alpha_{n+1}\left(c+z_{n+1}\right)=v \circ \alpha_{n+1}(c)$ для всех $c \in C_{n+1} \cap\left(C_{n+1}-z_{n+1}\right)$;

(A4) для любого $i, 0 \leqslant i<m_{b}$, найдется множество $C_{n+1, i} \subset C_{n+1}$, такое, что

$$
\begin{gathered}
C_{n+1, i}-2 h_{n} \subset C_{n+1}, \\
\alpha_{n+1}(c)=\alpha_{n+1}\left(c-2 h_{n}\right)+v^{i}(b) \quad \text { для всех } c \in C_{n+1, i}, \\
\left|\frac{\# C_{n+1, i}}{\# C_{n+1}}-\frac{1}{(k+1) m_{b}}\right|<\frac{2}{n m_{b}}
\end{gathered}
$$

(A5) найдется множество $C_{n+1, \Delta} \subset C_{n+1}$, такое, что

$$
\begin{gathered}
C_{n+1, \Delta}-2 h_{n}-1 \subset C_{n+1}, \\
\alpha_{n+1}(c)=\alpha_{n+1}\left(c-2 h_{n}-1\right) \quad \text { для всех } c \in C_{n+1, \Delta}, \\
\left|\frac{\# C_{n+1, \Delta}}{\# C_{n+1}}-\frac{k}{k+1}\right|<\frac{2}{n} .
\end{gathered}
$$

Таким образом, $C_{n+1}, h_{n+1}, z_{n+1}, \alpha_{n+1}$ полностью определены.

Теперь положим $F_{n}:=\left\{0,1, \ldots, h_{n}-1\right\}$. Обозначим $(C, F)$-конструкцию, отвечающую последовательности $\left(C_{n+1}, F_{n}\right)_{n \geqslant 0}$, через $(X, \mu, T)$. Так как из соотношения

$$
\# F_{n+1}=h_{n+1} \geqslant 2 r_{n} h_{n}=2 \# C_{n+1} \# F_{n}
$$


следует, что $\mu\left(X_{n+1}\right) \geqslant 2 \mu\left(X_{n}\right)$, то получаем $\mu(X)=\infty$.

Пусть $\mathscr{R}$ - хвостовая эквивалентность (или, иначе говоря, $T$-орбитальная эквивалентность) на $X$. Обозначим через $\alpha: \mathscr{R} \rightarrow K$ коцикл отношения $\mathscr{R}$, ассоциированный с последовательностью $\left(\alpha_{n}\right)_{n>0}$.

\section{§4. Эргодичность и кратная рекуррентность преобразований $T^{\times p} \times T_{\alpha}$}

Предложение 4.1. Преобразование $T^{\times p} \times T_{\alpha}$ эргодично для любого $p>0$.

Доказательство. Сначала докажем эргодичность преобразования $T^{\times p}$. Для простоты рассмотрим случай $p=2$. (Общий случай доказывается аналогично.) Заметим, что для каждого $n \in \mathscr{M}_{b, 1}-1$ найдутся множества $C_{n+1}^{\prime}$ и $C_{n+1}^{\prime \prime}$ в $C_{n+1}$, такие, что

(a) если $c \in C_{n+1}^{\prime}$, то $2 h_{n}+c \in C_{n+1}$,

(b) если $c \in C_{n+1}^{\prime \prime}$, то $2 h_{n}+1+c \in C_{n+1}$,

(c) $\# C_{n+1}^{\prime} \geqslant 1 / 3 \cdot \# C_{n+1}$ и $\# C_{n+1}^{\prime \prime} \geqslant 1 / 3 \cdot \# C_{n+1}$.

Пусть заданы произвольные $n>0$ и $f, f^{\prime}, d, d^{\prime} \in F_{n}$. Для определенности считаем, что $f \geqslant f^{\prime}$ и $d \geqslant d^{\prime}$. Положим $s:=\max \left(f-f^{\prime}, d-d^{\prime}\right)$. Найдем $k>n$, такое, что пересечение $\{n+1, \ldots, k\} \cap \bigsqcup_{b \in \mathscr{K}}\left(\mathscr{M}_{b, 1}-1\right)$ состоит из $s$ точек, скажем, $l_{1}, \ldots, l_{s}$. Положим $A:=f+\sum_{i=1}^{k} A_{i}$ и $B:=d+\sum_{i=1}^{k} B_{i}$, где

$$
A_{i}:=\left\{\begin{array}{ll}
C_{i}, & i \notin\left\{l_{1}, \ldots, l_{s}\right\}, \\
C_{i}^{\prime \prime}, & i \in\left\{l_{1}, \ldots, l_{f-f^{\prime}}\right\}, \\
C_{i}^{\prime}, & i \in\left\{l_{f-f^{\prime}+1}, \ldots, l_{s}\right\},
\end{array} \quad B:= \begin{cases}C_{i}, & i \notin\left\{l_{1}, \ldots, l_{s}\right\}, \\
C_{i}^{\prime \prime}, & i \in\left\{l_{1}, \ldots, l_{d-d^{\prime}}\right\}, \\
C_{i}^{\prime}, & i \in\left\{l_{d-d^{\prime}+1}, \ldots, l_{s}\right\} .\end{cases}\right.
$$

Из (а)-(с) несложно вывести, что

- $[A]_{k} \subset[f]_{n},[B]_{k} \subset[d]_{n}$;

- $\mu\left([A]_{k}\right) \geqslant \frac{1}{3^{s}} \mu\left([f]_{n}\right), \mu\left([B]_{k}\right) \geqslant \frac{1}{3^{s}} \mu\left([d]_{n}\right)$;

- $T^{2\left(h_{l_{1}-1}+\cdots+h_{l_{s}-1}\right)}[A]_{k} \subset\left[f^{\prime}\right]_{n}, T^{2\left(h_{l_{1}-1}+\cdots+h_{l_{s}-1}\right)}[B]_{k} \subset\left[d^{\prime}\right]_{n}$.

Осталось применить следующую лемму.

Лемма 4.2. Пусть фиксированы число $p>0$ и отображение $\delta: \mathbb{Z}^{p} \rightarrow \mathbb{R}_{+}$, причем $\sum_{g \in \mathbb{Z}^{p}} \delta(g)<1 / 2$. Если для каждого $n>0 u f_{1}, \ldots, f_{p}, f_{1}^{\prime}, \ldots, f_{p}^{\prime} \in F_{n}$ найдутся множество $A \subset\left[f_{1}\right]_{n} \times \cdots \times\left[f_{p}\right]_{n}$ и число $s \in \mathbb{Z}$, такие, что $T^{\times p} A \subset$ $\left[f_{1}^{\prime}\right]_{n} \times \cdots \times\left[f_{p}^{\prime}\right]_{n} u$

$$
\mu(A)>\delta\left(f_{1}-f_{1}^{\prime}, \ldots, f_{p}-f_{p}^{\prime}\right) \mu\left(\left[f_{1}\right]_{n} \times \cdots \times\left[f_{p}\right]_{n}\right),
$$

то преобразование $T^{\times p}$ эргодично.

Лемма 4.2 является частным случаем леммы 2.4 из [7] или леммы 5.2 из [11].

Теперь проверим, что произведение $T^{\times p} \times T_{\alpha}$ является эргодическим. Представим это преобразование в виде косого произведения $\left(T^{\times(p+1)}\right)_{1 \otimes \alpha}$. Для заданных $n>0, f_{1}, \ldots, f_{p+1} \in F_{n}$ и $a \in \mathscr{K}$ можно найти число $k>n$, такое, что $k+1 \in \mathscr{N}_{a}$. Положим

$$
\begin{gathered}
A_{i}:=f_{i}+C_{n+1}+\cdots+C_{k}+C_{k+1,0}, \\
A:=\left[A_{1}\right]_{k+1} \times \cdots \times\left[A_{p}\right]_{k+1} \subset\left[f_{1}\right]_{n} \times \cdots \times\left[f_{p}\right]_{n} .
\end{gathered}
$$


Из условия (А2) предыдущего параграфа вытекает, что

$$
\begin{gathered}
\left(T^{\times p}\right)^{-2 h_{k}}\left[A_{1}\right]_{k+1} \times \cdots \times\left[A_{p}\right]_{k+1} \subset\left[f_{1}\right]_{n} \times \cdots \times\left[f_{p}\right]_{n}, \\
\frac{\mu^{p}(A)}{\mu^{p}\left(\left[f_{1}\right]_{n} \times \cdots \times\left[f_{p}\right]_{n}\right)}>\left(\frac{1}{2 m_{a}}\right)^{p},
\end{gathered}
$$

а равенства

$$
1 \otimes \alpha\left(x,\left(T^{\times p}\right)^{-2 h_{k}} x\right)=\alpha\left(x_{p+1}, T^{-2 h_{k}} x_{p+1}\right)=a
$$

выполняются для всех $x=\left(x_{1}, \ldots, x_{p+1}\right) \in A$. Так как $\mathscr{K}$ плотно в $K$, из стандартного критерия эргодичности для коциклов [27] получим, что $1 \otimes \alpha$ эргодичен, т. е. преобразование $T^{\times p} \times T_{\alpha}$ эргодическое.

Замечание 4.3. Преобразование $T^{\times p}$ является кратно рекуррентным для любого $p>0$, что следует из замечания 2.4(i) из [11]. Из [15] вытекает, что преобразование $T^{\times p} \times T_{\alpha}$ также обладает этим свойством.

\section{§5. Доказательство основного результата}

Так как случай $E=\{1\}$ тривиален (каждое преобразование ранга 1, сохраняющее бесконечную меру, имеет простой спектр), предположим, что $E \neq\{1\}$. Зафиксируем натуральное число $m$, такое, что $m+1 \in E$. Наша иель - показать, что $\mathscr{M}\left(T^{\odot m} \times T_{\alpha, H}\right)=E$, где объекты $T, \alpha, K, H$ были определены в $\S 3$.

Лемма 5.1. Пусть $a, b \in \mathscr{K}$. Тогда для каждого $\chi \in \widehat{K}$,

(i) $U_{T, \chi}^{2 h_{n}} \rightarrow l_{\chi}(a) \cdot I$ nрu $\mathscr{N}_{a}-1 \ni n \rightarrow \infty$;

(ii) $U_{T, \chi}^{2 h_{n}} \rightarrow \frac{l_{\chi}(b)}{k+1} \cdot I+\frac{k}{k+1} U_{T, \chi}^{*} n p u \mathscr{M}_{b, k}-1 \ni n \rightarrow \infty$.

Доказательство. Докажем только п. (ii), поскольку п. (i) доказывается аналогично. Пусть $n+1 \in \mathscr{M}_{b, k}$.

Рассмотрим произвольное множество $A \subset F_{n}$. Замечаем, что $[A]_{n}=[A+$ $\left.C_{n+1}\right]_{n+1}$. Поэтому из условия (А4) (см. §3) вытекает, что для любого $x \in T\left[F_{n}\right]_{n}$ имеют место равенства

$$
\begin{aligned}
U_{T, \chi}^{2 h_{n}} 1_{[A]_{n}}(x)= & \sum_{i=0}^{m_{b}-1} \chi\left(\alpha\left(T^{2 h_{n}} x, x\right)\right) 1_{\left[A+C_{n+1, i}\right]_{n+1}}\left(T^{2 h_{n}} x\right) \\
& +\chi\left(\alpha\left(T^{2 h_{n}} x, x\right)\right) 1_{\left[A+C_{n+1, \Delta}\right]_{n+1}}\left(T^{2 h_{n}} x\right)+\bar{o}(1) \\
= & \sum_{i=0}^{m_{b}-1} \chi\left(v^{i}(b)\right) 1_{\left[A+C_{n+1, i}-2 h_{n}\right]_{n+1}}(x) \\
& +\chi\left(\alpha\left(T^{-1} x, x\right)\right) 1_{\left[A+C_{n+1, \Delta}-2 h_{n}-1\right]_{n+1}}\left(T^{-1} x\right)+\bar{o}(1) \\
= & \sum_{i=0}^{m_{b}-1} \chi\left(v^{i}(b)\right) 1_{\left[A+C_{n+1, i}-2 h_{n}\right]_{n+1}}(x) \\
& +U_{T, \chi}^{*} 1_{\left[A+C_{n+1, \Delta}-2 h_{n}-1\right]_{n+1}}(x)+\bar{o}(1) .
\end{aligned}
$$


Таким образом,

$$
U_{T, \chi}^{2 h_{n}}-\sum_{i=0}^{m_{b}-1} \chi\left(v^{i}(b)\right) 1_{\left[C_{n+1, i}-2 h_{n}\right]_{n+1}}-U_{T, \chi}^{*} 1_{\left[C_{n+1, \Delta}-2 h_{n}-1\right]_{n+1}} \rightarrow 0
$$

при $\mathscr{M}_{b, k}-1 \ni n \rightarrow \infty$. Здесь функции $1_{\left[C_{n+1, i}-2 h_{n}\right]_{n+1}}$ и $1_{\left[C_{n+1, \Delta}-2 h_{n}-1\right]_{n+1}}$ рассматриваются как операторы умножения (ортопроекции) в $L^{2}(X, \mu)$. Осталось воспользоваться неравенствами (A4), (А5) и тем фактом, что для любой последовательности $C_{n}^{\prime} \subset C_{n}$ при условии, что $\# C_{n}^{\prime} / \# C_{n} \rightarrow \delta$, мы имеем слабую сходимость

$$
1_{\left[C_{n}^{\prime}\right]_{n}} \rightarrow \delta I, \quad n \rightarrow \infty .
$$

Доказательство теоремы 0.1. Из предложения 4.1 следует, что преобразование $T^{\odot m} \times T_{\alpha, H}$ эргодично.

Имеет место естественное разложение оператора $U_{T \odot m} \times T_{\alpha, H}$ в ортогональную прямую сумму,

$$
U_{T \odot m} \times T_{\alpha, H}=\bigoplus_{\chi \in \widehat{K / H}}\left(U_{T}^{\odot m} \otimes U_{T, \chi}\right) .
$$

Докажем следующие утверждения:

(i) $U_{T}^{\odot m} \otimes U_{T}$ имеет однородный спектр кратности $m+1$,

(ii) $U_{T}^{\odot m} \otimes U_{T, \chi}$ имеет простой спектр, если $\widehat{K / H} \ni \chi \neq 0$,

(iii) $U_{T}^{\odot m} \otimes U_{T, \chi}$ и $U_{T}^{\odot m} \otimes U_{T, \xi}$ унитарно эквивалентны, если $\chi$ и $\xi$ принадлежат одной $\widehat{v}$-орбите,

(iv) меры максимального спектрального типа операторов $U_{T}^{\odot m} \otimes U_{T, \chi}$ и $U_{T}^{\odot m} \otimes$ $U_{T, \xi}$ взаимно сингулярны, если $\chi$ и $\xi$ не принадлежат одной $\widehat{v}$-орбите.

Из равенства $\mathscr{M}\left(U_{T}^{\odot m} \otimes U_{T}\right)=\mathscr{M}\left(\left(U_{T}\right)_{\mathfrak{S}_{m}}^{\otimes(m+1)}\right)$ и леммы 1.1 получаем (i). По лемме 5.1(ii) имеет место сходимость

$$
U_{T}^{2 h_{n}} \rightarrow \frac{1}{k+1} I+\frac{k}{k+1} U_{T}^{*}
$$

при $\mathscr{M}_{b, k}-1 \ni n \rightarrow \infty$ для всех $b \in \mathscr{K}$ и $k \in \mathbb{N}$. Из доказательства леммы 2.2 следует, что унитарный оператор $U_{T}^{\odot(m+1)}$ имеет простой спектр. Теперь п. (i) получается из следствия 1.2 .

Так как $T$ является преобразованием ранга 1 (см, например, [25] для определения ранга), а отображение $[f] \ni x \mapsto \alpha(T x, x) \in K$ постоянно для каждого $f \in F_{n} \backslash\left\{h_{n}-1\right\}, n \in \mathbb{N}$, оператор $U_{T, \chi}$ имеет простой спектр. Если $\widehat{K / H} \ni \chi \neq 0$, то по лемме 2.1 (ii) найдется такое $b \in \mathscr{K}$, что $l_{\chi}(b) \neq 1$.

Пункт (ii) леммы 5.1 приводит к сходимости

$$
U_{T, \chi}^{2 h_{n}} \rightarrow \frac{l_{\chi}(b)}{k+1} I+\frac{k}{k+1} U_{T, \chi}^{*}
$$

при $\mathscr{M}_{b, k}-1 \ni n \rightarrow \infty, k=1, \ldots, m$. Теперь (ii) следует из (5.1) и (5.2) и из леммы 2.2 .

TaK как

$$
\sum_{n>0} \frac{\#\left(C_{n} \triangle\left(C_{n}-z_{n}\right)\right)}{\# C_{n}}=\sum_{n>0} \frac{2}{n^{2}}
$$


из леммы 3.1 вытекает, что преобразование $S_{\bar{z}}(X, \mu)$ корректно определено формулой (3.1), причем $S_{\bar{z}} \in C(T)$. Из условий (A1) и (А 3$)$ получаем, что условие (3.2) выполнено. Следовательно, из леммы 3.1 получаем, что

$\alpha \circ S_{\bar{z}}$ когомологичен $v \circ \alpha$,

и п. (iii) следует из замечания 2.3.

Пусть характеры $\chi, \xi \in \widehat{K}$ не принадлежат одной $\widehat{v}$-орбите. По лемме 2.1(ii) найдется $a \in \mathscr{K}$, для которого $l_{\chi}(a) \neq l_{\xi}(a)$. Из леммы 5.1(i) теперь получаем

$$
\left(U_{T}^{\odot m} \otimes U_{T, \chi}\right)^{2 h_{n}} \rightarrow l_{\chi}(a) I \quad \text { и } \quad\left(U_{T}^{\odot m} \otimes U_{T, \xi}\right)^{2 h_{n}} \rightarrow l_{\xi}(a) I
$$

при $n \rightarrow \infty$ и $n \in \mathscr{N}_{a}-1$. Это доказывает утверждение (iv). Из (i)-(iv) вытекает, что

$$
\mathscr{M}\left(U_{T \odot m \times T_{\alpha, H}}\right)=E .
$$

Авторы благодарят рецензента за замечания.

\section{ЛитерАтУРА}

[1] J. Aaronson, An Introduction to Infinite Ergodic Theory, Amer. Math. Soc., Providence, RI, 1997.

[2] T. Adams, N. Friedman, C. E. Silva, Rank one power weak mixing nonsingular transformations, Ergodic Theory Dynam. Systems, 21:5 (2001), 1321-1332.

[3] O. N. Ageev, On ergodic transformations with homogeneous spectrum, J. Dynam. Control Systems, 5:1 (1999), 149-152.

[4] O. N. Ageev, The homogeneous spectrum problem in ergodic theory, Invent. Math., 160:2 (2005), 417-446.

[5] O. N. Ageev, Mixing with staircase multiplicity function, Ergodic Theory Dynam. Systems, 28:6 (2008), 1687-1700.

[6] А. М. Вершик, И. П. Корнфельд, Общая эргодическая теория групп преобразований с инвариантной мерой. Глава 4. Периодические аппроксимачии и их применения. Эргодические теоремы. Спектральная и энтропийная теория общих групn преобразований, в кн.: Динамические системы-2. Итоги науки и техники. Современные проблемы математики. Фундаментальные направления, т. 2, ВИНИТИ, М., 1985, 70-89.

[7] A. I. Danilenko, Funny rank one weak mixing for nonsingular Abelian actions, Israel J. Math., 121 (2001), 29-54.

[8] A. I. Danilenko, Explicit solution of Rokhlin's problem on homogeneous spectrum and applications, Ergodic Theory Dynam. Systems, 26:5 (2006), 1467-1490.

[9] A. I. Danilenko, $(C, F)$-actions in ergodic theory, in: Geometry and Dynamics of Groups and Spaces, Progr. Math., vol. 265, Birkhauser, Basel, 2008, 325-351.

[10] A. I. Danilenko, On new spectral multiplicities for ergodic maps, Studia Math., 197:1 (2010), 57-68.

[11] A. I. Danilenko, C. E. Silva, Multiple and polynomial recurrence for Abelian actions in infinite measure, J. London Math. Soc., 69:1 (2004), 183-200.

[12] A. I. Danilenko, C. E. Silva, Ergodic theory: non-singular transformations, in: Encyclopedia of Complexity and Systems Science, Springer-Verlag, 2009, 3055-3083.

[13] H. Furstenberg, Recurrence in Ergodic Theory and Combinatorial Number Theory, Princeton University Press, Princeton, NJ, 1981.

[14] G. R. Goodson, J. Kwiatkowski, M. Lemańczyk, P. Liardet, On the multiplicity function of ergodic group extensions of rotations, Studia Math., 102:2 (1992), 157-174. 
[15] K. Inoue, Isometric extensions and multiple recurrence of infinite measure preserving systems, Israel J. Math., 140 (2004), 245-252.

[16] A. del Junco, A simple map with no prime factors, Israel J. Math., 104 (1998), 301320.

[17] S. Kakutani, W. Parry, Infinite measure preserving transformations with mixing, Bull. Amer. Math. Soc., 69 (1963), 752-756.

[18] A. B. Katok, Combinatorial constructions in ergodic theory and dynamics, University Lecture Series, vol. 30, Amer. Math. Soc., Providence, RI, 2003.

[19] A. Katok, M. Lemańczyk, Some new cases of realization of spectral multiplicity function for ergodic transformations, Fund. Math., 206 (2009), 185-215.

[20] J. Kwiatkowski (jr), M. Lemańczyk, On the multiplicity function of ergodic group extensions. II, Studia Math., 116:3 (1995), 207-215.

[21] В. И. Оселедец, О спектре эргодических автоморфизмов, Докл. АН. СССР, 168 (1966), 1009-1011.

[22] E. A. Robinson, Ergodic measure-preserving transformations with arbitrary finite spectral multiplicities, Invent. Math., 72:2 (1983), 299-314.

[23] E. A. Robinson, Transformations with highly nonhomogeneous spectrum of finite multiplicity, Israel J. Math., 56 (1986), 75-88.

[24] V. V. Ryzhikov, Transformations having homogeneous spectra, J. Dynam. Control Systems, 5:1 (1999), 145-148.

[25] В. В. Рыжиков, Слабые предель степеней, простой спектр симметрических произведений и перемешивающие конструкции ранга 1, Матем. сб., 198:5 (2007), 137-159.

[26] В. В. Рыжиков, Спектральные кратности и асимптотические операторные свойства действий с инвариантной мерой, Матем. сб., 200:12 (2009), 107-120.

[27] K. Schmidt, Cocycles on ergodic transformation groups, Macmillan Lectures in Math., vol. 1, Macmillan Company of India, Delhi, 1977.

[28] А. М. Степин, Спектралъные свойства типичных динамических систем, Изв. AH CCCP, сер. матем., 50:4 (1986), 801-834.

ФТИНТ НАНУ им. Б. И. Веркина, Харьков e-mail: alexandre.danilenko@gmail.com

Поступило в редакцию

МГУ им. М. В. Ломоносова

25 мая 2009 г.

e-mail: vryzh@mail.ru 